CORRECTION

https://doi.org/10.1038/s41586-019-0907-y

\title{
Publisher Correction: Magnetic and magnetic inverse spin Hall effects in a non-collinear antiferromagnet
}

Motoi Kimata, Hua Chen, Kouta Kondou, Satoshi Sugimoto, Prasanta K. Muduli, Muhammad Ikhlas, Yasutomo Omori, Takahiro Tomita, Allan. H. MacDonald, Satoru Nakatsuji \& Yoshichika Otani

Correction to: Nature https://doi.org/10.1038/s41586-018-0853-0, published online 16 January 2019.

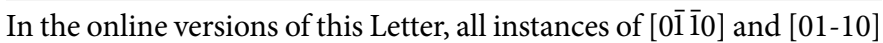
should have been [0110]. The original Letter has been corrected online. 